\title{
Análisis de la tendencia de la mortalidad por cirrosis hepática en Chile: Años 1990 a 2007
}

\author{
FAUSTINO TOMÁS ALONSO ${ }^{1}$, MARÍA LUISA GARMENDIA ${ }^{1}$, \\ MAGDALENA DE AGUIRRE ${ }^{2, a}$, JAVIER SEARLE ${ }^{2, a}$
}

\section{Mortality trend from liver cirrhosis in Chile from 1990 to 2007}

Background: There is a worldwide tendency towards a reduction in the rates of deaths due to cirrhosis. In Chile, a decrease in the number of hospital admissions due to this disease has been recorded. Aim: To assess general characteristics and temporal evolution of liver cirrhosis mortality in Chile between 1990 and 2007. Material and Methods: National death records and population databases were reviewed. Crude and age-adjusted mortality rates for alcoholic and non-alcoholic cirrhosis were calculated, evaluating their evolution in the study period and the relative risk by gender. Results: In the study period, 44,894 deaths caused by cirrhosis were recorded. Mortality rate was 16.6 deaths per 100,000 inhabitants. 54\% of deaths were attributed to non-alcoholic cirrhosis. There was a reduction in mortality rates for both types of cirrhosis. Males accounted for 83 and 65\% of deaths caused by alcoholic and nonalcoholic cirrhosis, respectively. The figures for relative risk of death were 5 and 1.9, respectively. Conclusions: Alcoholic cirrhosis was the preponderant cause among liver cirrhosis deaths. A decrease in mortality rates was observed in the study period. Improvements in disease treatment and control could possibly explain this trend.

(Rev Med Chile 2010; 138: 1253-1258).

Key words: Liver cirrhosis; Mortality; Public health.

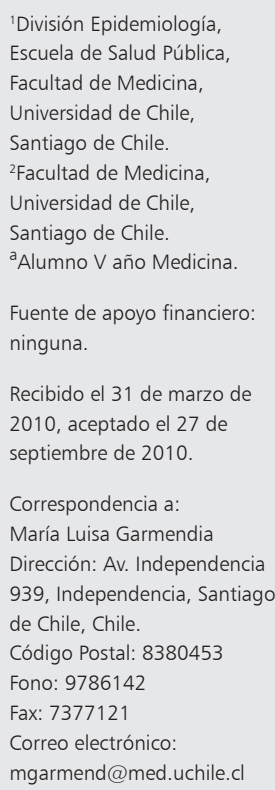

L a cirrosis hepática constituye un importante problema de salud alrededor del mundo, formando parte de las diez primeras causas de muerte ${ }^{1}$.

Bajo la clasificación de cirrosis hepática como causa de muerte subyacen dos grupos, el primero relacionado con el consumo de alcohol y otro grupo cuya etiología es variada, dentro de las cuales destacan la infección por VHB y VHC y aquellas asociadas a patología autoinmune, entre otras.

El estudio de Bosetti, mostró que en la década 1980-89, las tasas más altas de mortalidad por cirrosis en el mundo, se registraban en México y Chile. En este estudio, el análisis temporal reveló una tendencia a la disminución de estas tasas en la mayoría de los países del mundo ${ }^{2}$. En Chile, estudios anteriores al año 2000 mostraron una tendencia a la disminución de la mortalidad. Estos estudios postulan que este descenso pudo ser atribuido a una disminución en el número de casos o a un mejor abordaje terapéutico ${ }^{3-5}$.

En Chile no existen registros de morbilidad por cirrosis hepática, aunque las hospitalizaciones han mostrado una tendencia a la disminución tanto en la cirrosis de causa alcohólica, como no alcohólica. El análisis de las bases de egresos hospitalarios del Departamento de Estadísticas e Información en Salud (DEIS) del Ministerio de Salud evidenció que, entre los años 2001 y 2006, la tasa de hospitalización por 100.000 habitantes disminuyó de 
7,4 a 3,9 para cirrosis alcohólica y de 17,8 a 9,5 para la no alcohólica ${ }^{6}$. En términos de carga de enfermedad, la cirrosis hepática representa en la actualidad, la tercera causa de años de vida perdidos por discapacidad en hombres ${ }^{7}$.

En relación a los factores de riesgo, la prevalencia de consumo anual de alcohol en Chile es muy elevada, mostrando una tendencia al alza desde 1994, con prevalencias en los últimos años de alrededor de $57 \%$ en la población entre 15 y 65 años $^{8}$.

Dada la magnitud del problema, en términos de impacto sobre la salud pública nacional, surge la necesidad de contar con estudios sobre la situación epidemiológica actual de la cirrosis hepática. Este estudio pretende evaluar la mortalidad por esta enfermedad en Chile entre los años 1990 al 2007, en cuanto a su evolución temporal y características demográficas.

\section{Materiales y Métodos}

Se realizó un estudio ecológico de serie temporal para evaluar la tendencia y características de la mortalidad por cirrosis hepática tanto alcohólica como no alcohólica desde 1990 al 2007. Se revisaron la bases de defunciones del DEIS desde 1990 al 2007. Se seleccionaron todos aquellos casos en los cuales la causa básica de muerte fue cirrosis hepática alcohólica y cirrosis hepática no alcohólica. Los códigos utilizados para cirrosis hepática alcohólica fueron: 571.2 (CIE-9 hasta 1996) y K70.3 (CIE-10 a la fecha). Para cirrosis no alcohólica, se incluyó a la cirrosis biliar (código CIE-9: 571.6 hasta 1996 y códigos CIE-10: K74[3-5] a la fecha) y la cirrosis no alcohólica no especificada (código CIE-9: 571.5 hasta 1996 y código CIE-10: K74.6 a la fecha). Se registró el sexo, la edad en años al momento del fallecimiento y el año de defunción. Se calculó las tasas de mortalidad por cirrosis alcohólica y no alcohólica por 100.000 habitantes crudas y ajustadas por edad y sexo. Como denominador para las tasas crudas totales y por edad y sexo, se utilizaron las proyecciones de población del Instituto Nacional de Estadística (INE). El ajuste de las tasas se realizó mediante estandarización directa utilizando como población estándar la entregada por la Organización Mundial de la Salud para el año $2000^{9}$, estableciendo grupos de edad quinquenales hasta los 80 años y más. Dado el bajo número de defunciones en edades tenpranas, se agruparon las defunciones de 0 a 24 años y luego por decenios hasta los 75 años y más, de acuerdo a lo referido en la literatura. Se calculó el riesgo relativo de fallecer por cada una de estas causas, tanto para el sexo masculino, como para cada uno de los grupos etarios mencionados anteriormente. Se evaluó la tendencia de las tasas ajustadas de mortalidad mediante modelos de regresión de Prais-Winsten, con estimación robusta de la varianza, comprobando los supuestos y verificando la presencia de autocorrelación mediante el estadístico Durbin-Watson de los datos originales y corregidos ${ }^{10}$. Dicho modelo es similar a una regresión lineal, dado que entrega un coeficiente que expresa la magnitud de cambio de la tasa por cada año, pero corrigiendo la autocorrelación (autorregresión de primer orden) de los datos que suele existir en las series temporales. Se estimó el número de muertes que se redujeron anualmente, utilizando una población promedio de 7,5 millones de hombres y 8 millones de mujeres basada en la información de las proyecciones de población del INE. Los análisis estadísticos se realizaron con el software STATA $10.1^{11}$ y se estableció un nivel de significancia de $5 \%$.

\section{Resultados}

En Chile, entre 1990 y 2007 se registraron 44.894 defunciones por cirrosis hepática (3,1\% de las muertes totales en el período). De éstas, 24.126 $(53,74 \%)$ correspondieron a cirrosis hepática no alcohólica. Respecto al sexo, en la cirrosis hepática alcohólica, los hombres representaron el 83,14\% de las muertes, mientras que para cirrosis hepática no alcohólica el porcentaje fue menor, alcanzando $64,80 \%$. En relación a la edad al momento de la defunción, las muertes por causa alcohólica ocurrieron a una edad promedio de 57,68 años (D.E. $=11,95)$, inferior a la que se observó por cirrosis hepática de causa no alcohólica, cuyo promedio fue 62,3 años (D.E.=12,53). La tasa de mortalidad por cirrosis hepática para el período fue 16,60 muertes por 100.000 habitantes, 7,68 para la alcohólica y 8,92 para la no alcohólica.

Las tasas de mortalidad crudas y ajustadas, tanto en cirrosis alcohólica como no alcohólica, fueron más altas en hombres que en mujeres. En los hombres predominó la mortalidad por cirrosis alcohólica, a diferencia de las mujeres en las que se apreció una mayor mortalidad por cirrosis no alcohólica (Tabla 1). 
Tabla 1. Tasas de mortalidad por cirrosis hepática alcohólica y no alcohólica en Chile, 1990-2007

\begin{tabular}{|lcccccc|} 
& \multicolumn{2}{c}{ Alcohólica } & \multicolumn{2}{c}{ No alcohólica } & \multicolumn{2}{c|}{ Total } \\
& Cruda & Ajustada & Cruda & Ajustada & Cruda & Ajustada \\
Mujeres & 2,56 & 2,70 & 6,22 & 5,89 & 8,78 & 8,59 \\
Hombres & 12,91 & 15,09 & 11,39 & 13,42 & 24,30 & 28,51 \\
Total & 7,68 & 8,90 & 8,92 & 9,66 & 16,60 & 18,56 \\
\hline
\end{tabular}

Tasas por 100.000 habitantes.

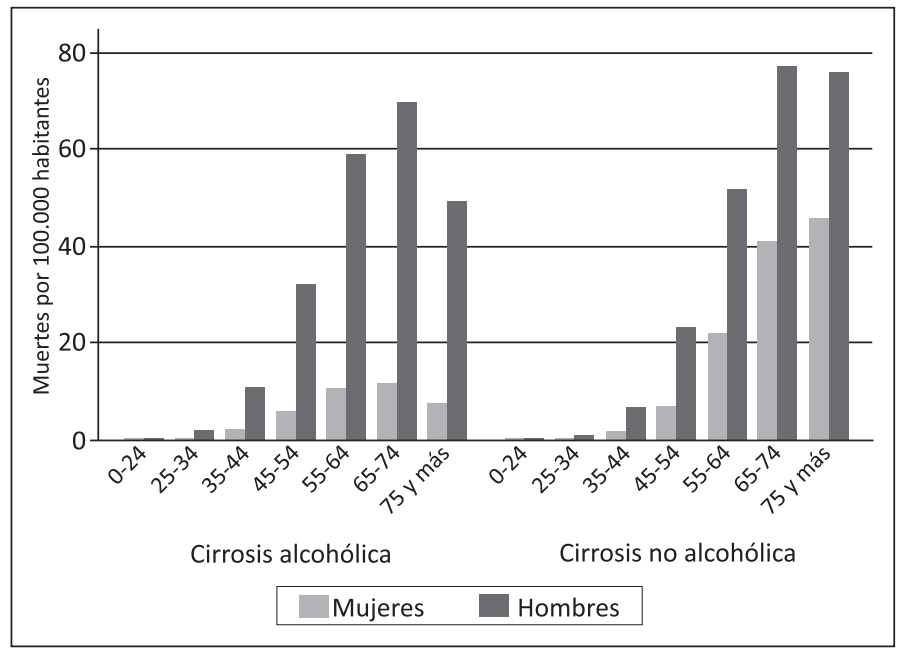

Figura 1. Tasas de mortalidad por cirrosis hepática en hombres y mujeres según tipo de cirrosis y grupo etario, Chile 1990-2007.
Con respecto a las tasas de mortalidad por grupos etarios, estas mostraron un aumento progresivo a medida que incrementa la edad hasta el grupo de 65 a 74 años que presentó la tasa más elevada, tanto para el total de defunciones $(95,77$ muertes por 100.000 habitantes), como para las defunciones por cirrosis alcohólica $(37,24$ por 100.000 habitantes). En cirrosis no alcohólica, la mayor tasa de mortalidad se observó en el grupo de 75 años o más (57,10 por 100.000 habitantes). Salvo en el grupo de 25 a 54 años, siempre las tasas de mortalidad fueron más altas para la cirrosis no alcohólica (Figura 1).

Se identificó el sexo masculino como factor de riesgo de morir por cirrosis en todos los grupos etarios y por ambas causas de cirrosis. Para cirrosis alcohólica, los hombres tuvieron un riesgo 5 veces mayor que las mujeres y el grupo etario de mayor riesgo fue el de los menores de 24 años, donde los hombres tenían un riesgo 7 veces mayor que el de las mujeres. En cambio, para cirrosis no alcohóli- ca, el riesgo de los hombres disminuyó a 1,88 y el grupo etario con la mayor diferencia de tasas fue el de 35 a 44 años, donde los hombres tuvieron un riesgo 3,5 veces mayor (Tabla 2).

Los modelos de regresión mostraron una tendencia a la disminución de las tasas de mortalidad ajustadas por edad, a través de los años, tanto en hombres como en mujeres y para ambas causas (Figura 2). La mayor disminución se observó en los hombres que fallecieron por cirrosis hepática alcohólica (coeficiente $=-0,91$ ), es decir, por cada año la tasa se redujo en 0,91 muertes por 100.000 habitantes (alrededor de 68 muertes menos cada año). En las mujeres, la disminución fue más baja que en los hombres (coeficiente $=-0,22$ ), pero también estadísticamente significativa, lo que se traduce que en promedio fallecieron 18 mujeres menos cada año.

También se encontró un descenso significativo en la tendencia de las muertes por causa no alcohólica, pero de menor magnitud. Para los hombres 
Tabla 2. Riesgo relativo de los hombres de fallecer por cirrosis hepática alcohólica y no alcohólica según grupo etario, Chile 1990-2007

\begin{tabular}{|cccc|}
\hline Grupo de edad (en años) & Alcohólica & No alcohólica & Total \\
\hline $0-24$ & 7,27 & 1,06 & 1,57 \\
\hline $25-34$ & 6,06 & 3,46 & 4,71 \\
\hline $55-44$ & 5,23 & 3,53 & 4,41 \\
\hline $45-54$ & 5,48 & 3,28 & 4,27 \\
\hline $55-64$ & 5,48 & 2,36 & 3,39 \\
$65-74$ & 6,00 & 1,88 & 2,79 \\
\hline 5 y más & 6,62 & 1,66 & 2,35 \\
\hline Total & 5,04 & 1,88 & 2,80 \\
\hline
\end{tabular}

Se utilizó como grupo de referencia a las mujeres.

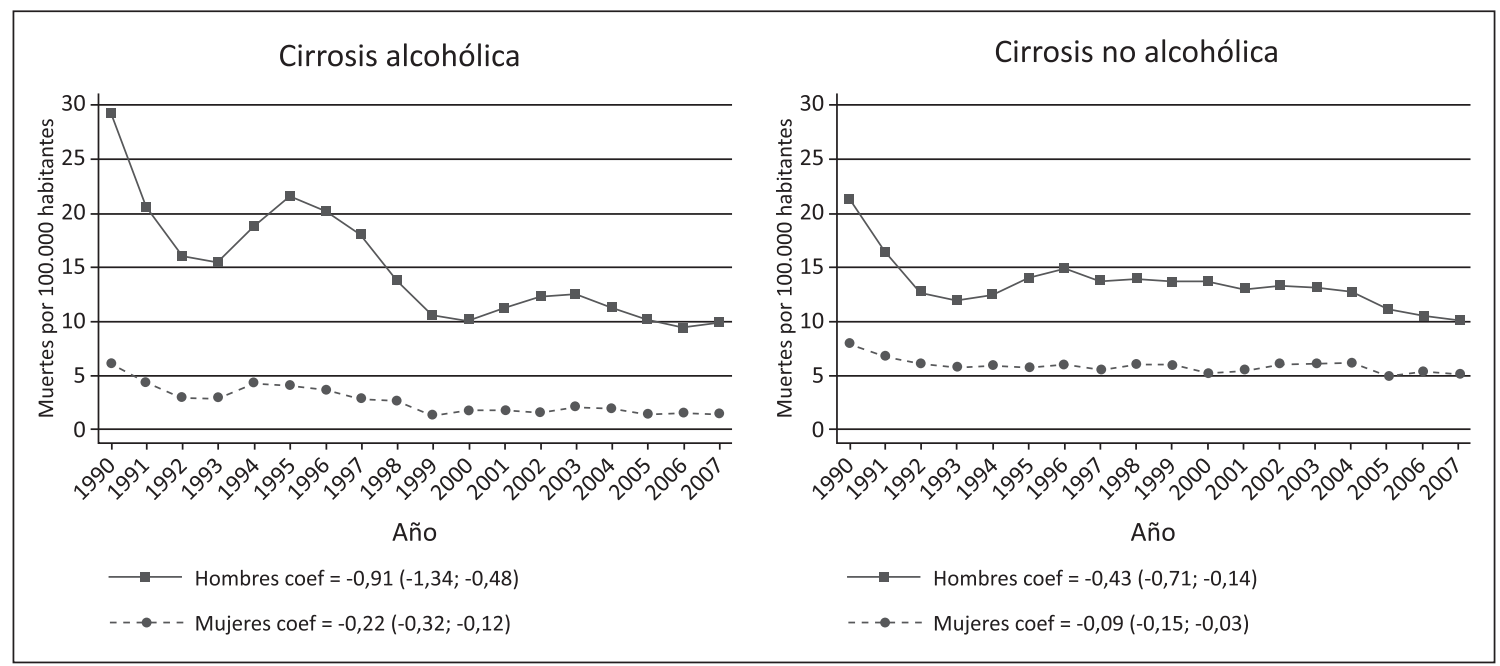

Figura 2. Evolución temporal de la mortalidad por cirrosis hepática alcohólica y no alcohólica según sexo, Chile 1990 - 2007. (Tasas estandarizadas por edad).

el coeficiente fue - 0,43 (32 muertes menos anualmente) y $-0,99$ para las mujeres (equivalente a 7 muertes menos por año).

\section{Discusión}

Esta actualización en el estudio de la mortalidad por cirrosis hepática en Chile mostró una tendencia a la disminución de las muertes atribuidas a esta causa. Este hallazgo es similar a lo reportado previamente por estudios nacionales ${ }^{5}$ y concordante con lo descrito por publicaciones internacionales ${ }^{2,12}$.

El descenso en las tasas a nivel internacional se ha relacionado a varios factores, entre ellos se mencionan las mejoras en el tratamiento de la cirrosis y sus complicaciones, incluyendo la ligadura y escleroterapia de várices esofágicas y gástricas, progresos en su farmacoterapia, el desvío portosistémico a través de un "stent" intrahepático colocado por vía transyugular (TIPS) y el trasplante hepático. Por otro lado, la disminución en la prevalencia de infección por virus de la hepatitis B y C, 
también ha sido relacionada a la disminución de la mortalidad en países como Italia y Japón ${ }^{2}$. Países como Estados Unidos de Norteamérica, Australia y otros en el sur de Europa han atribuido parte de esta disminución a los cambios en la cantidad, tipo y patrones de consumo de alcohol. Así, el consumo de bebidas de alto grado alcohólico o de manera excesiva ha sido asociado al aumento de la mortalidad $^{12-16}$. Esta explicación no parece ser la causa del descenso de las tasas en nuestro país, puesto que la prevalencia de consumo de alcohol en Chile ha registrado un aumento en los últimos años ${ }^{8}$, por lo que el descenso en la mortalidad podría atribuirse más bien a un mejor enfrentamiento terapéutico. Sin embargo, no contamos con series históricas de consumo de alcohol anteriores a 1994, o descripción de los patrones de éste que permitan dar una mayor solidez a esta aseveración.

También hay que considerar que no es posible descartar un subdiagnóstico como causa de esta disminución, aunque los registros de mortalidad han aumentado su calidad progresivamente disminuyendo el porcentaje de muertes mal clasificadas ${ }^{17}$.

Nuestro estudio encontró que los hombres tienen un riesgo tres veces mayor de fallecer por cirrosis hepática, similar a lo reportado por Medina para el período $1975-1999^{5}$. Este riesgo fue diferente al analizarlo por tipo de cirrosis, siendo mucho mayor en las de causa alcohólica, concordante a las diferencias en la prevalencia de consumo de alcohol entre hombres y mujeres ${ }^{8}$. Aunque en menor magnitud, en la cirrosis no alcohólica, los hombres también presentaron un riesgo mayor, posibles explicaciones de este hallazgo pueden residir en diferencias en la prevalencia de infección por virus hepatitis $\mathrm{C}$ y $\mathrm{B}$, ya que el mayor riesgo se presenta en hombres entre 25 y 54 años. Sin embargo, no es posible descartar que exista un error de clasificación en la causa de muerte dada la estigmatización asociada al consumo problemático de alcohol.

Nuestro estudio encontró una gran diferencia en la magnitud de los riesgos entre cirrosis alcohólica y no alcohólica en ciertos grupos etarios, especialmente en los extremos. Esto podría ser atribuido al reducido número de defunciones observadas en estos grupos, produciendo tasas lábiles.

En conclusión, aunque probablemente la disminución de la mortalidad por cirrosis hepática puede ser atribuida a un mejor manejo terapéutico, el alza en la prevalencia de consumo por alcohol podría revertir esta tendencia. Junto con los progresos terapéuticos, es necesario avanzar en estrategias de prevención y control de los factores de riesgo.

\section{Referencias}

1. Méndez-Sánchez N, Villa AR, Zamora-Valdés D, Morales-Espinosa D, Uribe M. Worldwide mortality from cirrhosis. Ann Hepatol 2007; 6: 194-5.

2. Bosetti C, Levi F, Lucchini F, Zatonski WA, Negri E, La Vecchia C. Worldwide mortality from cirrhosis: an update to 2002. J Hepatol 2007; 4 6(5): 827-39.

3. Medina E, Kaempffer AM. Epidemiología de la cirrosis hepática en Chile. Rev Med Chile 1974; 102 (6): 466-76.

4. Medina E, Kaempffer AM. Cirrosis hepática en Chile: consideraciones epidemiológicas. Rev Med Chile 1993; 121: 1324-31.

5. Medina E, Kaempffer AM. Cirrosis hepática en Chile. Revista Chilena de Salud Pública 2002; 6 (1).

6. Gobierno de Chile, Ministerio de Salud, Departamento de Estadísticas e Información en Salud. Base de egresos hospitalarios 2001-2006. Disponible en: http:Ildeis. minsal.cl. [Consultado el 10 de noviembre de 2009].

7. Gobierno de Chile, Ministerio de Salud, Departamento de Epidemiología. Estudio Carga De Enfermedad Y Carga Atribuible 2007. 2008.

8. Gobierno de Chile, Ministerio del Interior, Consejo Nacional para el Control de Estupefacientes. Estudio Nacional de Drogas en Población General de Chile 1994 a 2008.

9. Ahmad OB, Boschi-Pinto C, López AD, Murray CJ, Lozano R, Inoue M. Age standardization of rates: a new WHO standard. Ginebra: OMS; 2001.

10. Prais SJ, Winsten. CB. Trend Estimators and Serial Correlation. Cowles Commission Discussion Paper No 383. Chicago 1954.

11. StataCorp. Stata Statistical Software: Release 10. College Station. TX: StataCorp LP; 2007.

12. La Vecchia C, Levi F, Lucchini F, Franceschi S, Negri E. Worldwide patterns and trends in mortality from liver cirrhosis, 1955 to 1990. Ann Epidemiol 1994; 4: 480-6.

13. Ponicki WR, Gruenewald PJ. The impact of alcohol taxation on liver cirrhosis mortality. J Stud Alcohol 2006; 67: 934-8.

14. Gruenewald PJ, Ponicki WR. The relationship of alcohol sales to cirrhosis mortality. J Stud Alcohol 1995; 56: 63541.

15. Ramstedt M. Per capita alcohol consumption and liver 
cirrhosis mortality in 14 European countries. Addiction. 2001; 96 Suppl 1: S19-33.

16. Dalmau-Bueno A, García-Altes A, Mari-Dell'olmo M, Pérez K, Espelt A, Kunst AE, et al. Trends in socioeconomic inequalities in cirrhosis mortality in an urban area of Southern Europe : A multilevel approach. J Epidemiol Community Health. 2009 Oct 19.

17. Núñez FM, Icaza NM. Calidad de las Estadísticas de Mortalidad en Chile, 1997-2003. Rev Med Chile 2006; 134 (9): 1191-6. 